\title{
A variant form of $h M T H 1$, a human homologue of the $E$ coli mutT gene, correlates with somatic mutation in the p53 tumour suppressor gene in gastric cancer patients
}

\author{
Y Kimura, S Oda, A Egashira, Y Kakeji, H Baba, Y Nakabeppu, Y Maehara
}

J Med Genet 2004;41:e57 (http://www.jmedgenet.com/cgi/content/full/41/5/e57). doi: 10.1136/jmg.2003.013268

O xidative damage to diverse physiological molecules, including proteins, lipids, and nucleic acids, is an inevitable outcome of various cellular activities in living organisms. In particular, some oxidised forms of nucleotides cause miscoding of genetic information, and have therefore been present as a major threat for cells. Multigene systems to counteract such oxidative damage have evolved in diverse organisms. In $E$ coli, several mutants designated 'mutator' have been isolated, and in these cells the mutation rate is significantly elevated, due to disruption of genes regulating the spontaneous mutation rate on the genome. Previous studies using these mutators have identified three genes that function in the system to counteract mutagenic oxidative damage. The $m u t T^{-}$strain is one of the mutators that exhibit the highest spontaneous mutation rate. Maki $\mathrm{H}$ et al have shown that the product of the mutT gene hydrolyses an oxidised form of guanine nucleotides, 8-oxo$2^{\prime}$-deoxyguanosine 5 '-triphosphate $\left(8\right.$-oxo-dGTP). ${ }^{1}$ 8-oxodGTP incorporated into the genome stably pairs with adenine as well as cytosine in the template strand, accumulation of this oxidised form of guanine nucleotides leads to an increase in base substitution mutations-that is, A:T to G:C and G:C to $\mathrm{T}: \mathrm{A}$ transversions. In the $\mathrm{mutT}^{-}$strain, the rate for $\mathrm{A}: \mathrm{T}$ to C:G transversion is indeed elevated 1000 fold over the wild type level. ${ }^{2}$ The other two genes that function in cooperation with mutT are mutM (fpg) and mutY, both of which encode a DNA glycosylase to excise deleterious bases on the genome. ${ }^{3}$ The former excises 8-oxo-guanine in the opposite site of cytosine on the genome, the latter removing adenine that pairs with 8-oxo-guanine. Thus, even in cells lacking MUT, $\mathrm{G}: \mathrm{C}$ to T:A transversions are suppressed low. ${ }^{4}$ Multiplicity of cellular anti-mutagenic systems guarantees the spontaneous mutation rate on the genome at an extremely low level.

Several mammalian counterparts of these $E$ coli genes are now known. MTHl is the first identified mammalian homologue of $E$ coli mutT. ${ }^{5}$ The human MTHI gene, hMTHl, has been described in detail. ${ }^{6}$ Altered function of $h M T H 1$ and consequent elevation of the mutation rate may be an attractive hypothesis for various human diseases, particularly cancer. In several human diseases, including some common malignancies, the nucleotide sequence of hMTHI has been explored. ${ }^{6}$ However, no apparent mutations were found. Instead, a single nucleotide polymorphism (SNP) at the first nucleotide of codon 83, which results in amino acid change from valine (V83: GTG) to methionin (M83: ATG), has been found, and the incidence of the M83 allele is relatively frequent in healthy controls (allele frequency $=0.09) .^{7}$ In mice, an mutT homologue has been identified and designated Mthl. MTH1-null mice, in which Mthl is homozygously disrupted, have been reported. ${ }^{8}$ Although the estimated increase in the mutation rate was only twice that in wild type counterparts, relatively frequent tumourigenesis was observed in the liver, lung, and stomach of the animals. ${ }^{9}$ In

\section{Key points}

- $h M T H 1$ is a human homologue of the E coli mutT gene, which encodes a polypeptide that hydrolyses a potent mutagenic substrate for DNA replication, 8-oxo-dGTP. Altered function of hMTHI has been suspected in various pathological states.

- In some common malignancies, the nucleotide sequence of hMTHI has been explored. However, no mutations have been found. Instead, $G: C$ to $A: T$ transition at the first nucleotide of codon 83, GTG(Val) to ATG(Met), has been reported as a polymorphism.

- We show here that this polymorphic variation (V83M) is significantly more frequent in gastric cancer patients than in healthy controls, and correlates with mutation in the p53 tumour suppressor gene.

- V83M variation of hMTH1 correlated with mutation in p53 suppressor gene. The frequency of p53 mutation is significantly higher in tumours harbouring the M83: ATG allele $(6 / 14,42.9 \%)$ than those without this variant allele $(9 / 58,15.5 \%)$.

- Connection between variant hMTH1 and mutation in a common tumour suppressor gene may suggest a possible mechanism for a mutator phenotype underlying the pathogenesis of tumours.

patients with liver and lung cancer, sequence of hMTHl has been determined. ${ }^{7}$ However, no relevant sequence alterations were found, and the M83 allele is not also frequent in patients with these malignancies.

Gastric cancer is one of the most common malignancies in human populations, and in this cancer mutations are frequently found in various genes, including proto-oncogenes and tumour suppressor genes, as in other cancers. ${ }^{10}$ It still remains controversial whether increase in mutation rate plays an important role in tumourigenesis, and the sources of mutations in cancer are unknown.

Increased occurrence of gastric tumours in MTHI-null mice prompted us to explore the structure of this gene in a patient population. Here we report that the minor polymorphic base alteration at codon 83 is significantly more frequent in gastric cancer patients than in healthy controls, and correlates with mutation in $p 53$ tumour suppressor gene.

\section{MATERIALS AND METHODS}

\section{Patients and tissue specimens}

Samples were collected from 72 gastric cancer patients who underwent surgery in our department from January 1990 to December 1997. Men and women numbered 47 and 25, 
respectively; age ranged from 35 to 86 years, with a mean of 64 years. Written informed consent for studies using the tissues was obtained from each patient. Ethical approval was obtained from the IRB of Kyushu University. Specimens were taken immediately after resection and placed in liquid nitrogen.

\section{Reverse transcription-polymerase chain reaction (RT- PCR) for hMTHI gene}

Total RNA was extracted from cancerous and corresponding normal tissue specimens using ISOGEN (Nippon Gene, Toyama, Japan), according to the manufacturer's instructions. The first strand of cDNA was synthesised using $2 \mu \mathrm{g}$ of total RNA, random hexadeoxynucleotide primer (TaKaRa, Tokyo, Japan), and RAV-2 reverse transcriptase (TaKaRa). After the first strand synthesis, PCR was performed using a GeneAmp 9600 thermal cycler (Applied Biosystems, Foster City, CA, USA.). A $50 \mu \mathrm{l}$ reaction mixture contained $0.2 \mu \mathrm{M}$ oligonucleotide primer, $25 \mathrm{ng}$ cDNA, I X PCR buffer (TaKaRa), $250 \mu \mathrm{M}$ dNTP, and $2.5 \mathrm{U}$ ExTaq polymerase (TaKaRa). The oligonucleotide primers used to amplify the hMTHI sequence were 5' -ACCTGCCCCACCAATTACA-3' (forward), and 5'-GCCTCTTGTAAAGACTGGTT-3' (reverse) (fig 1). The thermal conditions were as follows: 35 cycles at $95^{\circ} \mathrm{C}$ for three seconds, $55^{\circ} \mathrm{C}$ for 45 seconds, and $72^{\circ} \mathrm{C}$ for 60 seconds; and one cycle at $72^{\circ} \mathrm{C}$ for five minutes. The PCR products were purified using a microcon-100 microconcentrator (Amicon, Beverly, MA, USA.).

\section{Genomic PCR for p53 gene}

Genomic DNA was extracted from tumour specimens. Tissue was ground in liquid nitrogen and lysed in digestion buffer ( 10 mM Tris.Cl, pH 8.0; 0.1 M EDTA, pH 8.0; 0.5\% SDS; and $20 \mu \mathrm{g} / \mathrm{ml}$ pancreatic RNase). After treatment with proteinase $\mathrm{K}$ and extraction with phenol, DNA was precipitated with ethanol, and then dissolved in 1 X TE ( 10 mM Tris-Cl, pH 7.5; and $1 \mathrm{mM}$ EDTA). To amplify the sequences corresponding to p53 exon 5 to exon 9, PCR was carried out using a GeneAmp 2400 thermal cycler (Applied Biosystems). A $100 \mu \mathrm{l}$ of reaction mixture contained $100 \mathrm{ng}$ genomic DNA, $10 \mu \mathrm{M}$ of each primer, $200 \mu \mathrm{M}$ dNTP, and $2.5 \mathrm{U}$ Taq DNA polymerase (TaKaRa). The primer sequences were for exon 5: $5^{\prime}$-TCTGTTCACTTGTGCCCTGAC-3' (forward), and $5^{\prime}$ ATCAGTGAGGAATCAGAGGCC-3'(reverse); for exon 6: 5'GCGCTGCTCAGATAGCGATG-3'(forward), and 5'-GGAGGG CCACTGACAACCA-3'(reverse); for exon 7: 5'-TGCCACAG GTCTCCCCAAGG-3' (forward), and 5'-GCACAGCAGGCCA GTGTGCA-3'(reverse); for exons 8 and 9: 5'-TTGGGAGTA GATGGAGCCT-3' (forward), and 5'-AGTGTTAGACTGGAAA CTTT-3'(reverse). The thermal conditions were as follows: one cycle at $95^{\circ} \mathrm{C}$ for 10 minutes; 35 cycles at $95^{\circ} \mathrm{C}$ for 30 seconds, $62^{\circ} \mathrm{C}$ for 30 seconds, and $72^{\circ} \mathrm{C}$ for 45 seconds. PCR products were purified using a microcon-100 microconcentrator (Amicon).

\section{DNA sequencing}

Labelled dideoxynucleotide terminator cycle sequencing reactions were performed using ABI Prism Dye Terminator Sequencing Kits (Applied Biosystems) and an ABI Prism 310 Genetic Analyser (Applied Biosystems). For each of PCR products, the same primers were used as a sequencing primer. Data were analysed using the ABI sequence software, DNA Sequencing Analysis, ver. 3.0 (Applied Biosystems). In each analysis, electropherograms were checked visually, not to misread minute sequence alterations. Sequence alterations were designated by comparison with the data deposited in the NCBI database sequence alterations found in one PCR product were verified by reverse sequencing and finally

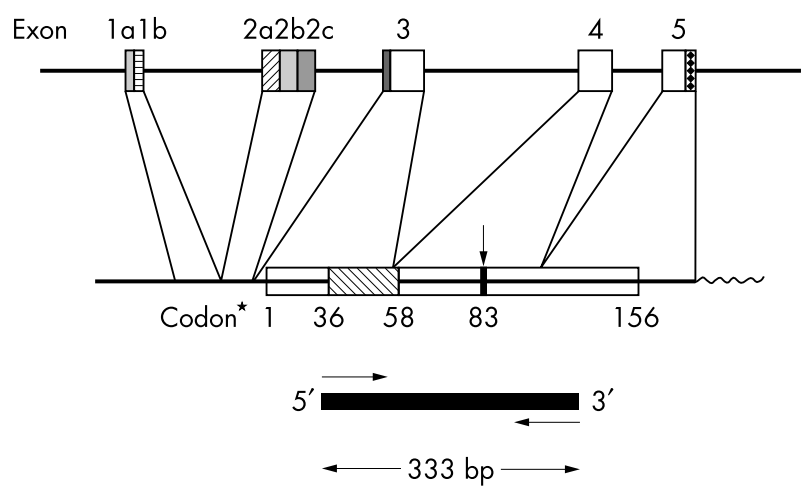

Figure 1 Schematic representation of $h M T H 1$ gene structure and sequencing strategy. Genomic and cDNA structures are shown. The position of codon 83 is indicated by a vertical arrow. The region corresponding to the 'phosphohydrolase module' is shown by a hatched box on mRNA. The 333-bp region, including codon 10-107, was amplified by RT-PCR. PCR primers are indicated by horizontal arrows. Another polymorphism in the $5^{\prime}$ untranslated region is also shown (asterisk). ${ }^{28}$

confirmed in more than two independently amplified PCR products.

\section{RESULTS}

A variant form of $h M T H I$ (M83) is more frequent in gastric cancer patients

In a panel of 72 gastric cancer patients, the nucleotide sequence of the hMTHl gene was determined using reverse transcript-polymerase chain reaction (RT-PCR) products. The 333-bp cDNA fragment that encompasses a region coding the 'phosphohydrolase module', the catalytic domain of oxidised purine nucleoside triphosphatase, was amplified and sequenced (fig 1). In agreement with previous reports, ${ }^{6}$ the minor M83 allele that encodes methionin (ATG), instead of valine (GTG), at codon 83 was found in several patients. According to the sequence data, the subject population was divided into three allelotypes, V83: GTG/V83: GTG, V83: GTG/ M83: ATG, and M83: ATG/M83: ATG (table 1). In gastric cancer patients, the M83 allele appeared more frequent than that in healthy controls $\left(\chi_{2}\right.$ test, $\left.\mathrm{p}=0.007\right)$. Among 72 gastric cancer patients, 14 (19.5\%) carried the M83 allele. Four patients $(5.6 \%)$ were homozygous for this variant allele. The subject population was next divided into two subgroups, one homozygous for this variant allele and the other. Another $\chi_{2}$ test revealed that the allelotype homozygous for the variant $h M T H 1$ allele (M83) was significantly more frequent in gastric cancer patients than in healthy controls $(\mathrm{p}=0.01)$ (table 1). Thus, we conclude that the variant form of $h M T H 1$ (M83) is more frequent in gastric cancer patients. These sequence alterations were also confirmed by sequencing PCR products of genomic DNA extracted from tumour specimens and corresponding normal tissues (data not shown). No other sequence alterations were found within the region examined.

We statistically tested whether this V83M variation of hMTHI correlates with clinicopathological features of the patients. However, no statistically significant correlation was found between the presence of this variant allele and any of the common clinicopathological features of patients (data not shown).

\section{V83M variation of $h M T H 1$ correlates with mutation in p53 gene}

hMTH1 encodes an enzyme that hydrolyses mutagenic oxidised purine nucleoside triphosphates such as 8-oxodGTP. Variation of a cellular component functioning in the 
Table 1 The frequency of single nucleotide polymorphism at codon 83 in the hMTHI gene: a comparison between gastric cancer patients and healthy controls

\begin{tabular}{llll}
\hline hMTH1 allelotype & Gastric cancer $(\mathbf{n}=\mathbf{7 2})$ & Controls $(\mathbf{n}=\mathbf{4 0 0})^{7}$ & \\
\hline V83: GTG/V83: GTG & $58(80.5 \%)$ & $330(83.5 \%)^{7}$ & \\
V83: GTG/M83: ATG & $10(13.9 \%)$ & $67(16.8 \%)^{7}$ & \\
M83: ATG/M83: ATG & $4(5.6 \%)$ & $3(0.8 \%)^{7}$ & $\mathrm{p}=0.0073$ \\
& & & \\
V83: GTG/V83: GTG, & $68(94.4 \%)$ & $397(99.2 \%)^{7}$ & \\
V83: GTG/M83: ATG & $4(5.6 \%)$ & $3(0.8 \%)^{7}$ & $\mathrm{p}=0.01$ \\
M83: ATG/M83: ATG & & & \\
& & & \\
\hline
\end{tabular}

anti-mutagenic systems may affect the sequence stability maintained throughout the genome. Carcinogenesis is thought to comprise stepwise alterations in proto-oncogenes or tumour suppressor genes. We therefore examined the relationship between $h M T H 1$ variation and mutation in $p 53$ gene, a tumour suppressor gene most commonly mutated in various human malignancies. In our panel of 72 patients with gastric cancer, $p 53$ mutations resulting in an amino acid substitution were detected in 15 tumours $(20.8 \%)$. The mutations were predominantly $\mathrm{G}: \mathrm{C}$ to $\mathrm{A}: \mathrm{T}$ transitions in codons, including the acknowledged hot spots (data not shown). Intriguingly, $p 53$ mutation correlated with the variant form of hMTHl (table 2). The frequency of $p 53$ mutation was significantly higher in tumours harbouring at least one M83 allele than in those without M83 allele (Fisher's exact test, $\mathrm{p}=0.034$ ). However, in a comparison between tumours homozygous for M83 allele and others, difference in the incidence of $p 53$ mutation was not statistically confirmed, probably due to a paucity of M83homozygous tumours. Among nine p53-mutant tumours without the M83 allele, G:C to A:T and A:T to G:C transitions were noted in three $(3 / 9,33.3 \%)$ and two $(2 / 9,22.2 \%)$, respectively. G:C to T:A transversion was also shown in three tumours $(3 / 9,33.3 \%)$ (table 3$)$. On the other hand, among six tumours with $p 53$ mutations that possessed at least the M83 allele, G:C to A:T transitions were observed in four $(66.7 \%)$ (table 3). However, this partiality of mutation to G:C to A:T transition in tumours harbouring a M83 allele was not statistically confirmed. A:T to C:G transversion was not found in neither.

\section{DISCUSSION}

Altered function of hMTHl has been suspected in various pathological states, particularly in tumourigenesis, in human bodies. However, in spite of efforts to explore $h M T H 1$ gene in various human diseases, ${ }^{6}$ no mutations have been found. Single nucleotide polymorphism (SNP) at codon 83, V83: GTG to M83: ATG, was not clearly associated with lung or liver cancers. ${ }^{7}$ However, in the present study, we have shown that in gastric cancer patients this polymorphic variation of hMTHI is significantly more frequent than in healthy

Table 2 Relationship between $p 53$ gene status and V83M variation of $h M T H 1$

\begin{tabular}{lll}
\hline & \multicolumn{2}{l}{$\mathbf{p 5 3}$ gene status } \\
\cline { 2 - 3 } hMTH1 allelotype & $\mathbf{w t}(\mathbf{n}=57)$ & $\mathbf{m}(\mathbf{n}=\mathbf{1 5})$ \\
\hline V83: GTG/V83: GTG & $49(84.5 \%)$ & $9(15.5 \%)$ \\
V83:GTG/M83:ATG, & $8(57.1 \%)$ & $6(42.9 \%)$ \\
M83: ATG/M83: ATG & & $p=0.034$ \\
\hline
\end{tabular}

controls. In this context, this is the first report that has shown a connection between V83M variation of hMTHI and human malignancies.

Mutations are found in various genes in tumours. One may presume a state with an elevated mutation rate in some steps of tumourigenesis. However, it still remains controversial whether a state with an elevated mutation rate plays an important role in tumourigenesis. Bodmer and colleagues ${ }^{11} 12$ pointed out that tumour cells that harbour mutations in tumour suppressor genes or proto-oncogenes can be selected merely by phenotypical advantage, without an elevated mutation rate. On the other hand, a line of evidences suggests an existence of a state with an elevated mutation rate in tumours, and this state is now referred to as 'mutator phenotype'. The critical role of mutator phenotype in tumourigenesis has been emphasised by Loeb and colleagues. $^{1314}$ Spontaneous mutation rate on the genome is invariably regulated. Previous studies using $E$ coli mutators suggest that there are several cellular systems, the failure of which will lead to a significant increase in the mutation rate.

The first example of mutator phenotype derived from a disruption of such cellular systems is defective DNA mismatch repair in a cancer prone syndrome, hereditary non-polyposis colorectal cancer (HNPCC). ${ }^{15} 16$ Deficiency in mismatch repair is also associated with other human malignancies. ${ }^{17}$ One may expect that the spontaneous mutation rate is elevated in mismatch repair defective cells. Indeed, in mismatch repair gene knock out mouse cells, the mutation rate was 15 -fold higher than in wild type cells. ${ }^{18}$ However, mutations in representative tumourigenic genes, such as $p 53$ and ras, were not found in mismatch repair defective human tumours. Instead, insertion/deletion mutation in repetitive sequences such as microsatellites was observed. This phenomenon is now referred to as 'microsatellite instability' (MSI), and the cellular phenotype with MSI has been designated 'microsatellite mutator phenotype' (MMP). ${ }^{19} 20$ The discrepancy between knock out animals and

Table 3 Spectra of p53 mutations in gastric tumours with and without the M83 hMTHI allele

\begin{tabular}{|c|c|c|}
\hline p53 mutation & $\begin{array}{l}\text { V83:GTG/ } \\
\text { V83:GTG }(n=9)\end{array}$ & $\begin{array}{l}\text { V83:GTG/M83:ATG, } \\
\text { M83:ATG/M83:ATG } \\
(n=6)\end{array}$ \\
\hline \multicolumn{3}{|l|}{ Transition } \\
\hline $\mathrm{G}: \mathrm{C} \rightarrow \mathrm{A}: \mathrm{T}$ & $3(33.3 \%)$ & $4(67.4 \%)$ \\
\hline $\mathrm{A}: \mathrm{T} \rightarrow \mathrm{G}: \mathrm{C}$ & $2(22.2 \%)$ & 0 \\
\hline \multicolumn{3}{|l|}{ Transversion } \\
\hline $\mathrm{G}: \mathrm{C} \rightarrow \mathrm{T}: \mathrm{A}$ & $3(33.3 \%)$ & $1(16.7 \%)$ \\
\hline $\mathrm{G}: \mathrm{C} \rightarrow \mathrm{C}: \mathrm{G}$ & 0 & 0 \\
\hline$A: T \rightarrow T: A$ & $1(11.1 \%)$ & $1(16.7 \%)$ \\
\hline $\mathrm{A}: \mathrm{T} \rightarrow \mathrm{C}: \mathrm{G}$ & 0 & 0 \\
\hline
\end{tabular}


human tumours may suggest an oversimplification in our discussions

The second example is failure in the systems counteracting mutagenic oxidative damage in cells. In E coli, MutT, MutY, and MutM cooperatively function to minimise the effects of an oxidised form of guanine nucleotides, 8-oxo-dGTP, in the pool and on the genome. ${ }^{4}$ Mammalian homologues of these proteins and mice in which their genes are homozygously disrupted have been reported..$^{91-23}$ As mentioned above, the failure in this system that is clearly associated with human diseases has not been found. However, in MTHI-null ES cells, the mutation rate is elevated twofold higher, compared with the wild type counterparts. ${ }^{9}$ Altered function of $h M T H 1$ may also lead to accumulated mutations in various genes in human cells.

Connection between V83M variation and mutation in other genes, particularly proto-oncogenes or tumour suppressor genes, has not thus far been addressed. This is the first report of a connection between the variant hMTHI and p53 mutation.

E coli MutT hydrolyses an oxidised nucleotide, 8-oxo-dGTP, into its monophosphate form. ${ }^{1} 8$-oxo-dGTP incorporated in the genome pairs with adenine, as well as cytosine, which results in $\mathrm{G}: \mathrm{C}$ to $\mathrm{T}: \mathrm{A}$ or $\mathrm{A}: \mathrm{T}$ to $\mathrm{C}: \mathrm{G}$ transversions. In an $E$ coli strain lacking MutT, the spontaneous occurrence of A:T to C:G transversion increases 1000-fold higher than in wild type strains. ${ }^{2}$ In the mutT $^{-}$strain, G:C to T:A transversions are suppressed low by compensatory activities of MutM and MutY glycosylases. ${ }^{4}$

Frequent variation of $h M T H 1$ in gastric cancer may suggest a biased spectrum in observed mutations. However, the majority of mutations found in $p 53$ was G:C to A:T transitions, albeit this bias has not been statistically confirmed (table 3). No A:T to C:G transversions were found. In fact, the biological significance of V83M variation has not been determined. Yakushiji $\mathrm{H}$ et $\mathrm{al}^{24}$ reported that recombinant M83-variant hMTHI exhibited an increased heat lability in both structure and catalytic function; however, within a physiological range of temperature, difference in the activity to hydrolyse 8-oxo-dGTP was not confirmed.

Recently, other forms of oxidised nucleotides have been found as a mutagenic substrate for DNA replication. 2hydroxy-2'-deoxyadenosine $5^{\prime}$-triphosphate (2-OH-dATP) is known to be formed in cells by an oxidative stress ${ }^{25}$ and, once incorporated into the genome, pairs with various bases including cytosine ${ }^{26}$ Pairing of cytosine with an incorporated adenine nucleotide results in $\mathrm{G}: \mathrm{C}$ to $\mathrm{A}: \mathrm{T}$ transitions. Intriguingly, hMTHl efficiently hydrolyses this oxidised adenine nucleotide as well as 8-oxo-dGTP, whereas $E$ coli MutT does only the latter. ${ }^{627}$ Difference in the activity to hydrolyse 2-OH-dATP between M83-variant hMTHl and its authentic form is now being approached in our laboratories.

V83M variation may conceal a previously unrecognised biological significance. $\mathrm{H}$ Oda et $a^{28}$ reported that the polymorphism at codon 83 is tightly linked with another SNP, exon $2 b(G T)$ to exon $2 b(G C)$ at the 5 '-splicing site of exon $2 \mathrm{~b}$ segment that is an exonic intron and spliced out by alternative splicing (fig 1). In the exon $2 b(G C)$ allele, an alternative translation initiation occurs on hMTHl transcripts, resulting in hMTHl, a protein that possesses a functional mitochondrial targeting sequence (Sakai and Nakabeppu, in preparation). Although this polymorphism has not been addressed in this study, a tight linkage between M83 allele and the exon $2 \mathrm{~b}(\mathrm{GC})$ allele may suggest the existence of another molecular variety with different biological functions. This present study suggests that a functional alteration of hMTHl due to these polymorphisms may occur in vivo, and that an imbalance among these molecular varieties may relate to tumourigenesis, at least in the stomach.

\section{ACKNOWLEDGEMENTS}

We are extremely grateful to Y Nakatsu, T Tsuzuki, and M Sekiguchi for helpful discussions. The expert assistance in DNA sequencing by $\mathrm{H}$ Baba, K Miyamoto, and Y Ikematsu are gratefully acknowledged, as are $\mathrm{K}$ Akazawa's comments on the statistical analyses.

\section{Authors' affiliations}

Y Kimura, A Egashira, Y Kakeji, H Baba, Y Maehara, Department of Surgery and Science, Graduate School of Medical Sciences, Kyushu University, Fukuoka, Japan

S Oda, Department of Pathology, Institute for Clinical Research, National Kyushu Cancer Center, Fukuoka, Japan

Y Nakabeppu, Department of Immunobiology and Neuroscience, Medical Institute of Bioregulation, Kyushu University, and CREST, JST, Fukuoka, Japan

This study was partly supported by a grant from the Ministry of Education, Science, Sports, and Culture of Japan.

Correspondence to: S Oda, Institute for Clinical Research, National Kyushu Cancer Center, Fukuoka 811-1395, Japan; soda@nk-cc.go.jp

\section{REFERENCES}

1 Maki H Sekiguchi M. MutT protein specifically hydrolyses a potent mutagenic substrate for DNA synthesis. Nature 1992;355:273-5.

2 Yanofsky C, Cox EC, Horn V. The unusual mutagenic specificity of an E. Coli mutator gene. Proc Natl Acad Sci U S A 1966;55:274-81

3 Michaels ML, Cruz C, Grollman AP, Miller JH. Evidence that MutY and MutM combine to prevent mutations by an oxidatively damaged form of guanine in DNA. Proc Natl Acad Sci U S A 1992;89:7022-5.

4 Tajiri T, Maki H, Sekiguchi M. Functional cooperation of MutT, MutM and MutY proteins in preventing mutations caused by spontaneous oxidation of guanine nucleotide in Escherichia coli. Mutat Res 1995:336:257-67.

5 Sakumi K, Furuichi M, Tsuzuki T, Kakuma T, Kawabata S, Maki H, Sekiguchi M. Cloning and expression of cDNA for a human enzyme that hydrolyzes 8-oxo-dGTP, a mutagenic substrate for DNA synthesis. J Biol Chem 1993;268:23524-30.

6 Nakabeppu Y. Molecular genetics and structural biology of human MutT homolog, MTH1. Mutat Res 2001;477:59-70

7 Oda H, Taketomi A, Maruyama R, Itoh R, Nishioka K, Yakushiii H, Suzuki T, Sekiguchi M, Nakabeppu Y. Multi-forms of human MTH1 polypeptides produced by alternative translation initiation and single nucleotide polymorphism. Nucleic Acids Res 1999;27:4335-43.

8 Tsuzuki T, Egashira A, Kura S. Analysis of MTH1 gene function in mice with targeted mutagenesis. Mutat Res $2001 ; 477: 71-8$.

9 Tsuzuki T, Egashira A, Igarashi $\mathrm{H}$, Iwakuma T, Nakatsuru $Y$, Tominaga $Y$, Kawate H, Nakao K, Nakamura K, Ide F, Kura S, Nakabeppu Y, Katsuki M Ishikawa T, Sekiguchi M. Spontaneous tumorigenesis in mice defective in the MTH1 gene encoding 8-oxo-dGTPase. Proc Natl Acad Sci U S A 2001;98:11456-61.

10 El-Rifai W, Powell SM. Molecular biology of gastric cancer. Semin Radiat Oncol 2002; 12:128-40.

11 Tomlinson I, Bodmer W. Selection, the mutation rate and cancer: ensuring that the tail does not wag the dog. Nat Med 1999:5:11-12.

12 Tomlinson IP, Novelli MR, Bodmer WF. The mutation rate and cancer. Proc Natl Acad Sci U S A 1996;93:14800-3.

13 Loeb LA. A mutator phenotype in cancer. Cancer Res 2001:61:3230-9.

14 Loeb LA, Loeb KR, Anderson JP. Multiple mutations and cancer. Proc Nat Acad Sci U S A 2003;100:776-81.

15 Fishel R, Lescoe MK, Rao MR, Copeland NG, Jenkins NA, Garber J, Kane M, Kolodner R. The human mutator gene homolog MSH2 and its association with hereditary nonpolyposis colon cancer. Cell 1993;75:1027-38. [Corrected erratum appears in Cell 1994;77(1):167.]

16 Leach FS, Nicolaides NC, Papadopoulos N, Liu B, Jen J, Parsons R, Peltomaki $P$, Sistonen $P$, Aaltonen LA, Nystrom-Lahti M, Guan X-Y, Zhang J, Meltzer PS, Yu J-W, Kao F-T, Chen DJ, Cerosaletti KM, Fournier REK, Todd S, Lewis T, Leach RJ, Naylor SL, Weissenbach J, Mecklin J-P, Jarvinen H, Petersen GM, Hamilton SR, Green J, Jass J, Watson P, Lynch HT, Trent JM, de la Chapelle A, Kinzler KW, Vogelstein B. Mutations of a mutS homolog in hereditary nonpolyposis colorectal cancer. Cell 1993;75:1215-25.

17 Arzimanoglou II, Gilbert F, Barber HR. Microsatellite instability in human solid tumors. Cancer 1998;82:1808-20.

18 Andrew SE, Reitmair AH, Fox J, Hsiao L, Francis A, McKinnon M, Mak TW, Jirik FR. Base transitions dominate the mutational spectrum of a transgenic reporter gene in MSH2 deficient mice. Oncogene 1997;15:123-9.

19 Perucho M. Cancer of the microsatellite mutator phenotype. Biol Chem 1996:377:675-84

20 Perucho $M$. Microsatellite instability: the mutator that mutates the other mutator. Nat Med 1996;2:630-1.

21 Klungland A, Rosewell I, Hollenbach S, Larsen E, Daly G, Epe B, Seeberg E, Lindahl T, Barnes DE. Accumulation of premutagenic DNA lesions in mice defective in removal of oxidative base damage. Proc Natl Acad Sci U S A 1999:96:13300-5. 
22 Minowa $O$, Arai $T$, Hirano $M$, Monden $Y$, Nakai S, Fukuda $M$, Itoh M, Takano H, Hippou Y, Aburatani H, Masumura K, Nohmi T, Nishimura S, Noda T. Mmh/Oggl gene inactivation results in accumulation of 8-hydroxyguanine in mice. Proc Natl Acad Sci U S A 2000;97:4156-61

23 Sakumi K, Tominaga Y, Furuichi M, Xu P, Tsuzuki T, Sekiguchi $M$, Nakabeppu Y. Oggl knockout-associated lung tumorigenesis and its suppression by Mth 1 gene disruption. Cancer Res 2003;63:902-5.

24 Yakushiii H, Maraboeuf F, Takahashi M, Deng ZS, Kawabata S Nakabeppu Y, Sekiguchi M. Biochemical and physicochemical characterization of normal and variant forms of human MTH1 protein with antimutagenic activity. Mutat Res 1997;384:181-94.
25 Kamiya $\mathrm{H}$ Kasai $\mathrm{H}$. 2-hydroxyadenine (isoguanine) as oxidative DNA damage: its formation and mutation inducibility. Nucleic Acids Symp Ser 1995:233-4.

26 Kamiya H, Kasai H. Formation of 2-hydroxydeoxyadenosine triphosphate, an oxidatively damaged nucleotide, and its incorporation by DNA polymerases. Steady-state kinetics of the incorporation. J Biol Chem 1995;270:19446-50.

27 Fujikawa K, Kamiya H, Yakushiji H, Fujii Y, Nakabeppu Y, Kasai H. The oxidized forms of dATP are substrates for the human MutT homologue, the hMTH1 protein. J Biol Chem 1999;274:18201-5.

28 Oda $\mathrm{H}$, Nakabeppu $\mathrm{Y}$, Furuichi M, Sekiguchi M. Regulation of expression of the human MTH1 gene encoding 8-oxo-dGTPase. Alternative splicing of transcription products. J Biol Chem 1997;272:17843-50. 